\title{
Colossal Grain Boundary Strengthening in Ultrafine Nanocrystalline Oxides
}

\author{
Dereck N. F. Muche ${ }^{a}$, John W. Drazin ${ }^{\mathrm{a}}$, John Mardinly ${ }^{\mathrm{b}}$, Sanchita Dey ${ }^{\mathrm{a}}$, Ricardo H. R. Castro, ${ }^{\mathrm{a},}$
}

${ }^{a}$ Department of Materials Science \& Engineering, University of California-Davis, One Shields

Avenue, Davis, California 95616,United States

${ }^{\mathrm{b}}$ LeRoy Eyring Center for Solid State Science, Arizona State University, Tempe, Arizona 85287-

1704, United States

* Corresponding author: Ph.: 1(530)752-3724; FAX: 1(530) 752-1031; rhrcastro@ucdavis.edu

\begin{abstract}
One of the most classic size-effects in materials is the increase in the strength and hardness as the grain size decreases. However, a practical low size limit for this so-called grain boundary strengthening has been extensively reported for both metals and ceramics. Here, it is demonstrated that this limit is not observed in fully dense nanocrystalline magnesium aluminate, where hardness increases from 17.2 GPa to $28.4 \mathrm{GPa}$ (surpassing sapphire hardness) when grain sizes are refined from $188 \mathrm{~nm}$ to $7.1 \mathrm{~nm}$, respectively. The increasing trend is proportional to the square root of the grain size, following the Hall-Petch relationship, reassuring that common weakening mechanisms described in nanocrystalline metals might not be present in ceramics. To achieve such small grain sizes in fully dense ceramics, a new processing technique is introduced, Deformable Punch Spark Plasma Sintering, DP-SPS, in which nanoparticles are sheared under high pressures $(\sim 2 \mathrm{GPa})$ during densification at moderate temperatures $\left(720-870^{\circ} \mathrm{C}\right)$. This inhibits grain growth due to the low processing temperatures and destabilizes/eliminate isolated residual pores, known to detrimentally affect mechanical behavior of ceramics. Noticeably, the sintered material showed high transparency in the visible spectrum, being reported as one of the hardest transparent oxide material to date.
\end{abstract}


Keywords. Magnesium aluminate, Hall-Petch, Spark Plasma Sintering, Ceramics, Nanocrystalline materials

Nanocrystalline materials show attractive properties such as superior strength and hardness. In principle, grain boundaries act as pins for dislocations due to the local repulsive stress forces opposing their movement. This causes dislocations pile ups along the boundaries during loading, such that for the material to fail, dislocation must accumulate to overcome the repulsive stress. As the density of nucleated dislocations inside grains remains constant, by decreasing the grain size, grain boundary (GB) area is increased, decreasing net dislocation population requiring higher stresses to cause permanent deformation, macroscopically observed as enhanced strength and hardness [1]. This so-called Hall-Petch (H-P) relationship has been reported to have a low limit grain size below which the mechanical properties follow an inverse scaling law, often referred to as "inverse H-P relation"[2-5]. While in metals this limit is reported in the grain size range of 3-20nm [3], in ceramics, sizes as high as $130 \mathrm{~nm}$ were proposed as low grain size limits [6], attributing it to enhanced Coble creep, or GB sliding for instance [7-9].

Although comprehensive for nanocrystalline metals, the picture of crystal plasticity by dislocation glide is inadequate for ceramics due to the required energy barrier for dislocation nucleation and/ or insufficient number of slip planes [10]. This suggests the existence of another mechanism responsible for the size-dependent hardness increase. Indeed, decreased flaw size and density are observed inside ceramic nano-grains due to the increased GB area, as predicted by Griffith's criterion [11]. Because micro-flaws are critical in the mechanical behavior of ceramics and glasses; this infers stronger grains at the nanoscale. The quasi-plasticity model thus assumes that the load induced deformation in ceramics is accommodated by grain shearing, breaking (and 
reforming) bonds at the GB regions. Therefore, a correlation between the strength of the GBs, their networking area, and the room temperature macro-mechanical behavior exists. Based on this, an increased GB network (small grains) would lead to more efficient intergranular cracking deflection, macroscopically representing increase in hardness. No low grain size limit would be expected but for when the grains are so extremely small that there is loss of long-range ordering and the material is mostly amorphous. Although very limited observations are consistent with this claim [12], this can be explained by the dependence of mechanical behavior also on processing-induced defects $[10,13,14]$.

This work explores the hypothesis that a finely processed nanocrystalline ceramic can present colossal hardness values when grains are refined, given that a low grain size limit for HP relation in ceramics is virtually non-existent. Great challenges lie in obtaining fully dense polycrystalline ceramics (defect-free) with grain sizes below $100 \mathrm{~nm}$ due to the competitive grain growth and porosity stability at the final stages of sintering [17]. Here, a novel pressure-assisted densification process that enables full densification without significant grain growth is reported. The process is named Deformable Punch Spark Plasma Sintering, DP-SPS, and explores many advantages of the Spark Plasma Sintering combined with a punch designed to allow high pressures $\approx 2 \mathrm{GPa}$ and intentionally deform at high temperatures. While the high pressures decrease the densification temperatures, the punch deformation transfers transversal momentum to the grains to promote grain sliding and eliminate residual porosities commonly observed in the final stage of sintering under uniaxial pressure [18-20] - see Figure S1 and Figure S2 for schematics.

To test the hypothesis, ultrafine $\mathrm{MgAl}_{2} \mathrm{O}_{4}$ nanoparticles were synthesized by coprecipitation and used as starting powder for sintering (details on Supplementary Data). The 
synthesized nanoparticles had crystallite sizes of $3.8 \pm 0.2 \mathrm{~nm}$ and specific surface area 274.34 $\pm 0.41 \mathrm{~m}^{2} / \mathrm{g}$. Processing conditions are described in Table 1 . Figure $\mathrm{S} 3$ shows the X-ray diffraction patterns for the sintered samples and initial powder. The spinel phase remained highly crystalline after sintering, without the formation of second phases. The crystallite sizes determined from whole profile fitting are listed in Table 1 . The samples prepared under $720.0^{\circ} \mathrm{C}$ for 4 min under 2.0 10-9 Pa showed grain size of $7.1 \pm 0.2 \mathrm{~nm}$. The other processing conditions lead to increment in grain size up to $188.3 \pm 62.5 \mathrm{~nm}$. Samples were labeled according to the respective grain sizes (Table 1). Densities for all samples were measured using Archimedes method and helium gas displacement and confirmed that the DP-SPS technique was able to deliver truly theoretical densities $3.58 \pm 0.01 \mathrm{~g} . \mathrm{cm}^{-3}$, independent on the sintering condition. Extensive scanning electron microscopy was performed to investigate the presence of pore in the sample and none could be found. Figure S4 shows Energy-dispersive X-ray spectrum on the sintered samples confirming absence of elemental impurities. Figure 1 shows a bright field scanning transmission electron microscopy (STEM) image for the MAS-14 sample showing the nanosized grains with a relatively narrow size distribution. The average grain size from a collection of images was $14.6 \pm 5.1 \mathrm{~nm}$, which is quite consistent with the sizes from the X-ray diffraction pattern fitting. Only the MAS-14 sample was studied on STEM due to the complexity of the sample preparation process for such small grain sizes.

Figure 2 shows the transmission spectrum for the MAS-14 sample evidencing absence of major absorption bands from ultraviolet to near infrared range. Up to $81 \%$ of visible light with wavelength of $700 \mathrm{~nm}$ is transmitted. This is consistent with the high density of the sample, as small fractions of residual porosity would compromise transmission. Sapphire data is plotted for comparison [21]. Vickers indentation tests were carried out to measure the microhardness (Hv) 
as a function of the grain sizes (details on Supplementary Data). MAS-07 showed hardness of $28.38 \pm 1.67 \mathrm{GPa}$, an unprecedented value that surpasses sapphire hardness. Figure 3 shows the compilation of data as a function of the square root of grain size along with data from the literature for the same composition $[12,22,23]$ and for sapphire single crystal for comparison [24]. No breakdown of H-P relation is observed down to the smallest tested size.

The hardness values suggest the weakening mechanisms observed in nanocrystalline metals are not present in $\mathrm{MgAl}_{2} \mathrm{O}_{4}$, implying the quasi-plasticity model is plausible for explaining nanocrystalline ceramic behavior, although additional studies are needed. Nevertheless, the achievement speaks for the importance of controlled processing to obtain reliable samples to understand size-related phenomena. It is relevant then to discuss why the proposed DP-SPS method can effectively deliver small grain sizes and full densities. External pressures have been extensively used to improve densification and inhibit grain growth in SPS $[25,26]$. The main mechanisms proposed to contribute to densification under pressure are particle rearrangement, plastic yielding, power-law creep, and diffusional densification [18, 27]. However, achieving truly full densities without compromising the grain size is a challenge because there is always an apparent "need" for grain growth in the final stage of sintering to eliminate residual porosity $[18,19]$. This can be linked to the thermodynamic metastability of isolated pores (referred to as "spherical shells" surrounding pores) [27-29]. Because the GBs intersect pores, the Young's relation must be obeyed. Once the contact angle meets the dihedral angle, the driving force for pore elimination is diminished $[28,30]$. When this happens, grain growth becomes a necessary condition to allow densification, since this would shift the contact angles from the dihedral angle value, and destabilize the pores [17, 29]. Alternatively to grain growth, DP-SPS uses transversal momentum (coming from the pronounced deformation of the 
punch, Figure S5) to shift the contact angle by GB sliding, reactivating densification without need for an associated grain growth.

Although only tested here for $\mathrm{MgAl}_{2} \mathrm{O}_{4}$ DP-SPS can provide a reliable venue to fabricate truly dense nanostructures (with very small sizes) for the exploration of not only mechanical but also other interphase induced physical properties [30].

\section{Acknowledgements}

National Science Foundation DMR Ceramics 1055504 and 1609781 are acknowledged. DM thanks CNPq 236631/2012-8 for scholarship.

\section{References}

[1] Meyers, M. A.; Mishra, A.; Benson, D. J., Prog. Mater. Sci. 51 (2006) 427.

[2] Masumura, R. A.; Hazzledine, P. M.; Pande, C. S., Acta Mater. 46 (1998) 4527.

[3] Wang, Y.; Zhang, J.; Zhao, Y., Nano Lett. 7 (2007) 3196.

[4] Hahn, H.; Padmanabhan, K. A., Philos. Mag. B 76 (1997) 559.

[5] Hahn, H.; Mondal, P.; Padmanabhan, K. A., Nanostruct. Mater. 9 (1997) 603.

[6] Ehre, D.; Chaim, R., J. Mater. Sci. 46 (2008) 6139.

[7] Chokshi, A. H.; Rosen, A.; Karch, J.; Gleiter, H., Scrip. Metall. 23 (1989) 1679.

[8] Schiotz, J.; Di Tolla, F. D.; Jacobsen, K. W., Nature 391 (1998) 561.

[9] Nieman, G. W.; Weertman, J. R.; Siegel, R. W., J. Mater. Res. 6 (1991) 10121027.

[10] Chan, H. M.; Lawn, B. R., J. Am. Ceram. Soc. 71 (1988) 29.

[11] Ovid'ko, I.; Sheinerman, A., Rev. Adv. Mater. Sci 29 (2011) 105.

[12] Wollmershauser, J. A.; Feigelson, B. N.; Gorzkowski, E. P.; Ellis, C. T.; Goswami, R.;

Qadri, S. B.; Tischler, J. G.; Kub, F. J.; Everett, R. K., Acta Mater. 69 (2014) 9. 
[13] Xu, H. H.; Wei, L.; Padture, N. P.; Lawn, B.; Yeckley, R., J. Mater. Sci. 30 (1995) 869.

[14] Lawn, B. R., J. Am. Ceram. Soc. 81 (1998) 1977.

[15] Valiev, R. Z.; Estrin, Y.; Horita, Z.; Langdon, T. G.; Zechetbauer, M. J.; Zhu, Y. T., JOM $58(2006) 33$.

[16] Valiev, R., Nature Mater. 3 (2004) 511.

[17] Kang, S.-J. L., Sintering: densification, grain growth and microstructure. ButterworthHeinemann: 2004.

[18] Chaim, R.; Marder, R.; Estournés, C.; Shen, Z., Adv. Appl. Ceram. 111 (2012) 280.

[19] Chaim, R.; Bar-Hama, O. R., Mater. Sci. Eng. A 527 (2010) 462.

[20] Chaim, R.; Levin, M.; Shlayer, A.; Estournès, C., Adv. Appl. Ceram. 107 (2008) 159.

[21] Ke, R.; Zhang, Y.; Zhou, Y.; Yin, Z., Optik 125 (2014) 1465.

[22] Krell, A.; Klimke, J.; Hutzler, T., J. Eur. Ceram. Soc. 29 (2009) 275.

[23] Krell, A.; Bales, A., Int. J. Appl. Ceram. Tech. 8 (2011) 1108.

[24] Krell, A., Mater. Sci. Eng. A 245 (1998) 277.

[25] Guillon, O.; Gonzalez-Julian, J.; Dargatz, B.; Kessel, T.; Schierning, G.; Räthel, J.; Herrmann, M., Adv. Eng. Mater. 16 (2014) 830.

[26] Hahn, H.; Gleiter, H., Scrip. Metall. 16 (1979) 3.

[27] Groza, J., J. Mater. Eng. Perform. 2 (1993) 283.

[28] Varela, J. A.; Whittemore, O.; Longo, E., Ceram. Int. 16 (1990) 177.

[29] Lange, F., J. Eur. Ceram. Soc. 28 (2008) 1509.

[30] Straumal, B.; Mazilkin, A.; Protasova, S.; Stakhanova, S.; Straumal, P.; Bulatov, M.; Schütz, G.; Tietze, T.; Goering, E.; Baretzky, B. Rev. Adv. Mater. Sci. 41 (2015) 61. 
Table 1. Description of the Samples, SPS Conditions and Characterization. The grain sizes for samples MAS-07 to MAS-38 were determined by XRD diffraction. *Sample MAS-14 had its grains also determined based on TEM images being $14.6( \pm 5.1) \mathrm{nm}$. **Grain size for sample MAS-188 was determined from SEM images only. Vickers hardness has been determined based on measurements of the diagonals from SEM micrographs of the indentations (see Supplementary Data for further information).

\begin{tabular}{ccccc}
\hline Sample & Sintering conditions & Annealing condition & Grain size (nm) & Hv $_{\mathbf{v}}(\mathbf{G P a})$ \\
\hline MAS-07 & $720^{\circ} \mathrm{C} / 2.0 \mathrm{GPa} / 4 \mathrm{~min}$ & $520^{\circ} \mathrm{C} / 6 \mathrm{~h}$, under $\mathrm{O}_{2}$ & $7.1( \pm 0.2)$ & $28.38( \pm 1.67)$ \\
\hline MAS-14 & $850^{\circ} \mathrm{C} / 1.4 \mathrm{GPa} / 5 \mathrm{~min}$ & $650^{\circ} \mathrm{C} / 6 \mathrm{~h}$, under $\mathrm{O}_{2}$ & $13.8( \pm 1.2)^{*}$ & $26.42( \pm 1.67)$ \\
\hline MAS-16 & $860^{\circ} \mathrm{C} / 1.4 \mathrm{GPa} / 5 \mathrm{~min}$ & $650^{\circ} \mathrm{C} / 6 \mathrm{~h}$, under $\mathrm{O}_{2}$ & $16.1( \pm 1.2)$ & $24.26( \pm 1.24)$ \\
\hline MAS-19 & $870^{\circ} \mathrm{C} / 1.4 \mathrm{GPa} / 5 \mathrm{~min}$ & $650^{\circ} \mathrm{C} / 6 \mathrm{~h}$, under $\mathrm{O}_{2}$ & $19.0( \pm 1.7)$ & $24.27( \pm 1.62)$ \\
\hline MAS-38 & $\begin{array}{r}870^{\circ} \mathrm{C} / 1.4 \mathrm{GPa} / 5 \mathrm{~min}, \\
950^{\circ} \mathrm{C} / 500 \mathrm{MPa} / 5 \mathrm{~min}\end{array}$ & $750^{\circ} \mathrm{C} / 6 \mathrm{~h}$, under $\mathrm{O}_{2}$ & $38.8( \pm 3.0)$ & $20.32( \pm 1.42)$ \\
\hline MAS-188 & $850^{\circ} \mathrm{C} / 1.4 \mathrm{GPa} / 5 \mathrm{~min}$ & $1200^{\circ} \mathrm{C} / 16 \mathrm{~h}$, under air & $188.3( \pm 62.5)^{* *}$ & $17.22( \pm 1.69)$ \\
\hline
\end{tabular}




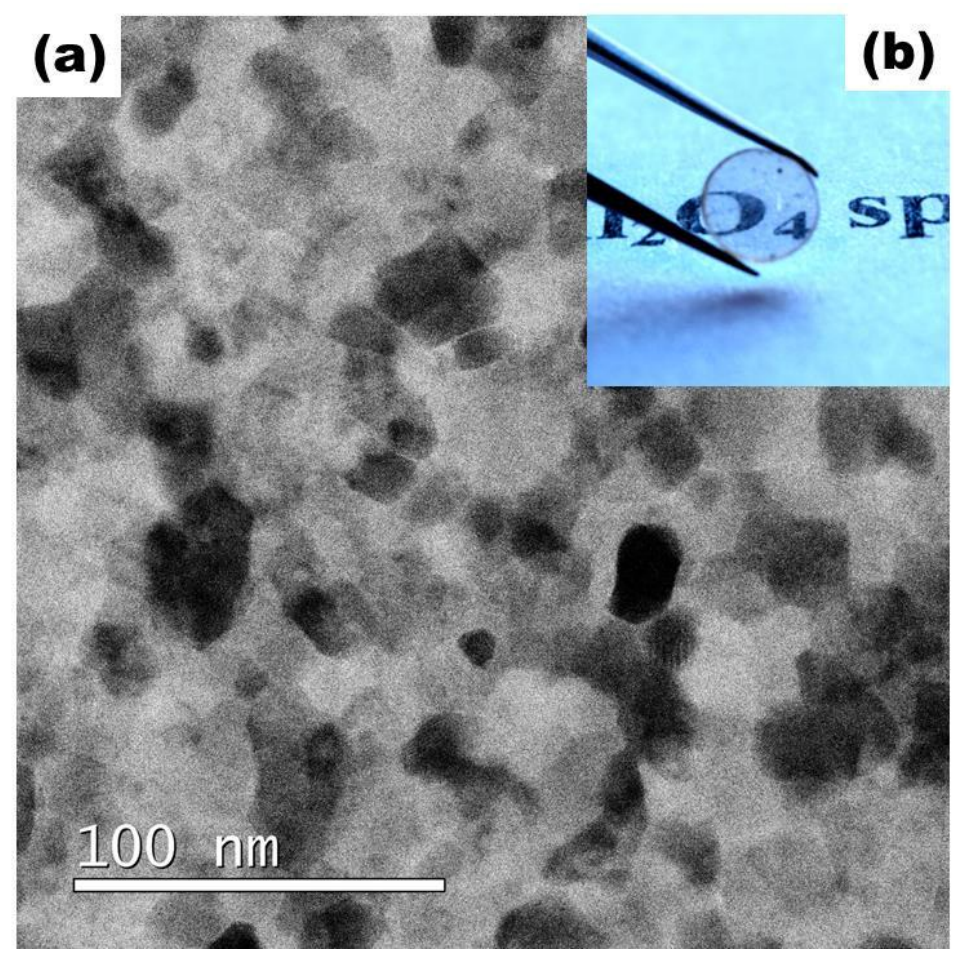

Figure 1. STEM image. (a) Bright field TEM image for sample A revealing an average grain size of $14.6( \pm 5.19) \mathrm{nm}$. (b) Picture of the sample MAS-14 elevated from background, showing high transparency in the visible range. 


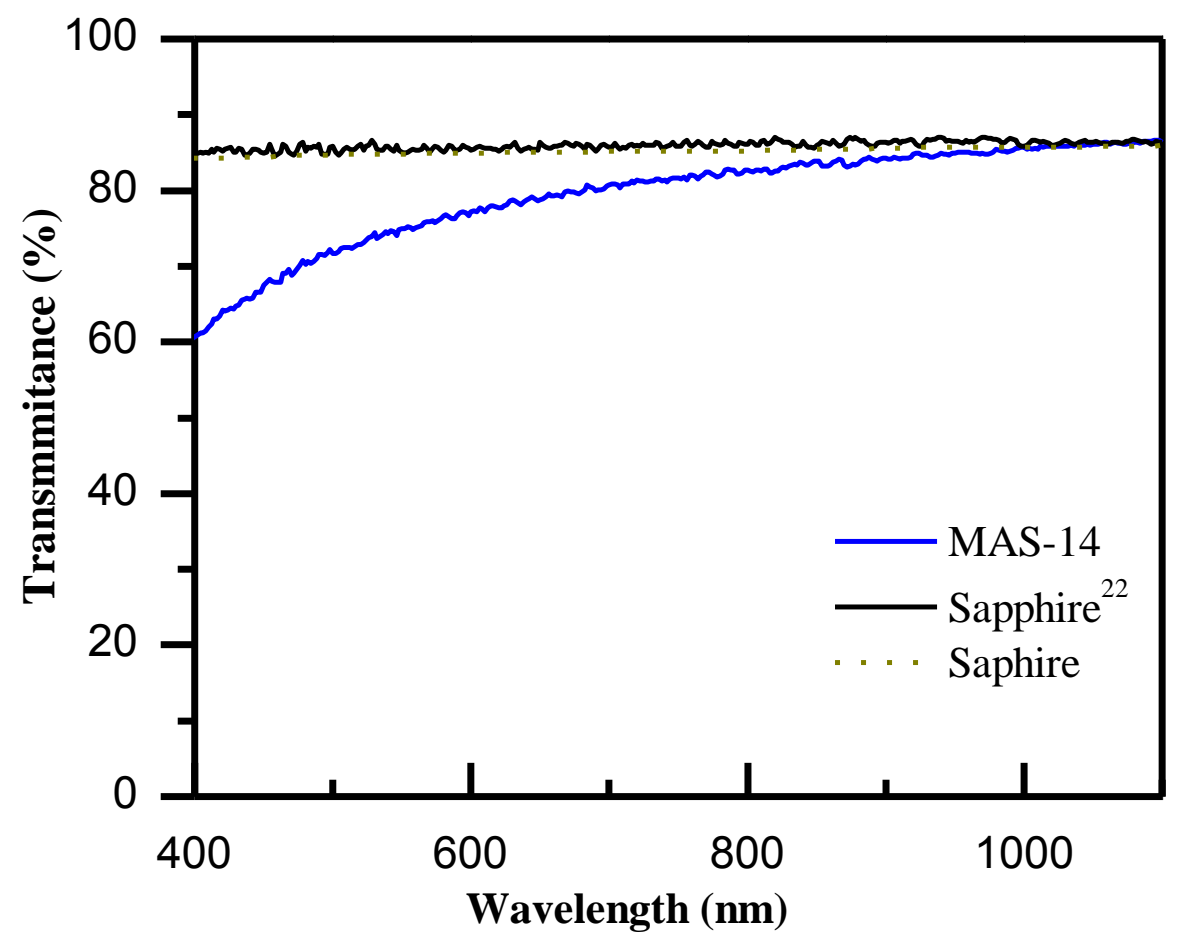

Figure 2. Optical transmissions. Transmission spectra obtained from MAS-14 sample (thickness of $0.92 \mathrm{~mm}$ ), compared to sapphire single crystal obtained experimentally using the same apparatus and from ref. 22. 


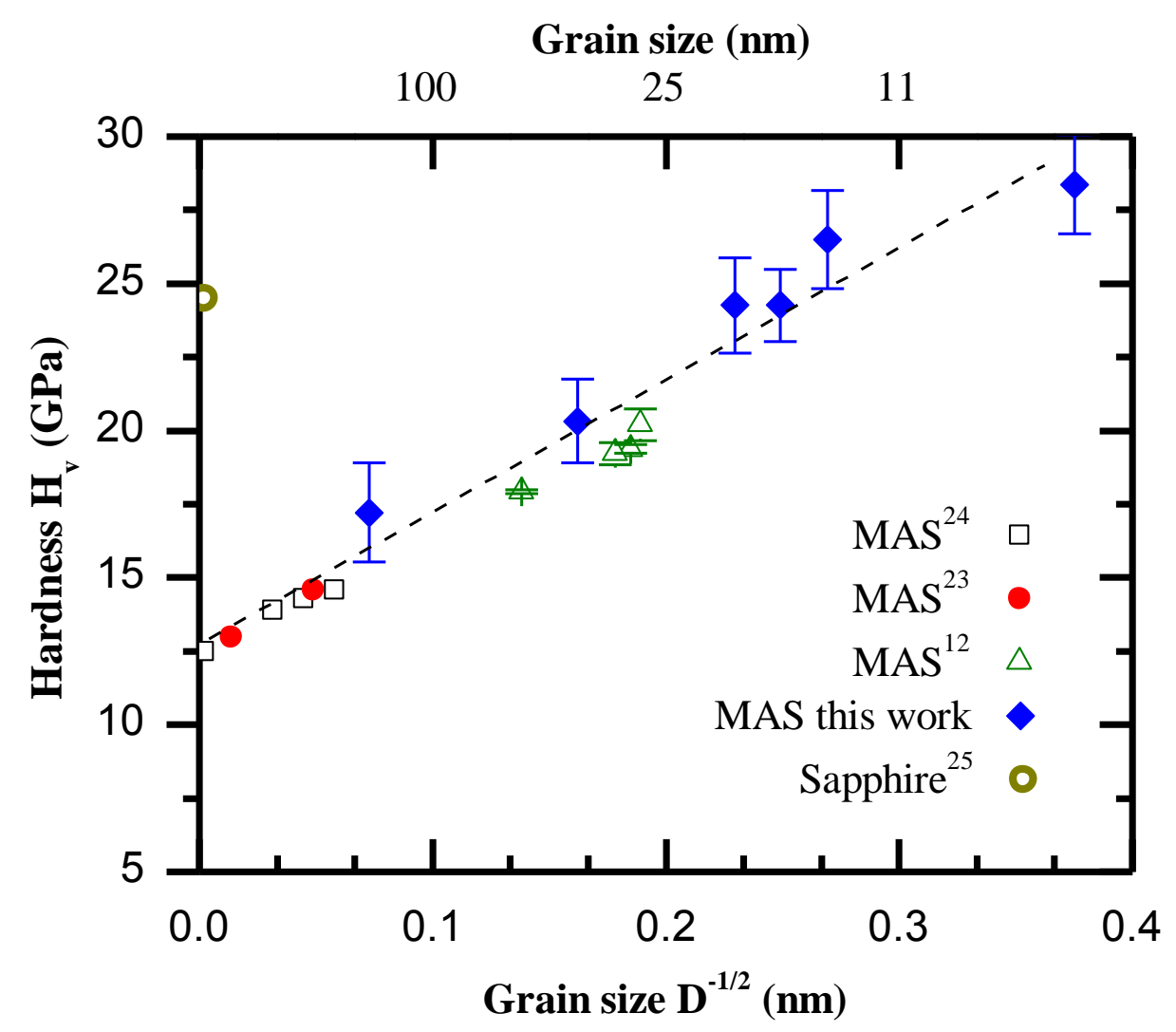

Figure 3. Hardness plot. Vickers hardness as function of square root of grain size for all sintered samples is represented. Literature data for $\mathrm{MgAl}_{2} \mathrm{O}_{4}$ (MAS) at different grains sizes are plotted as a comparison. Nanocrystalline MAS has restricted exploration with grain sizes no lower than $100 \mathrm{~nm}$ and no studies have been reported for grain sizes below 20nm. The dashed line represents the trend line for linear response of Hall-Petch relationship in terms of D-1/2. Hardness of some commercial materials such as gorilla glass and sapphire are also represented as comparison. 